\title{
Coronavirus \& diet: all about boosting immunity
}

\section{About COVID-1 9}

The world is currently experiencing the pandemic of Coronavirus $(\mathrm{CoV}-19)$. With the rising cases, proper nutrition and hydration are vital for stronger immune system and ultimately to fight against infectious disease. People need to understand that each nutrient and food plays a different role in the immune system, you cannot make hero of just one.It's a complex network of chemical compounds. Malnourishedindividuals are at greater risk of various bacterial or viral infections not just covid-19.Vitamin A, C, D, E, B6, B12, Folate, Iron, Zinc, Selenium \& copper are the key players in building up an immune system. When we talk about immunity building nutrients this doesn't mean that we have to take dietary supplements. You can easily fulfill your daily requirements by just keeping your plate COLORFUL. Below we will discuss how these requirements can be met through DIET ALONE!

Wonders of vitamin A: Most of the people need 700-900mcg of vitamin A daily which can easily be consumed by just eating a small sweet potato or a medium size carrot.You just have to make a colorful salad bowl and ENJOY!

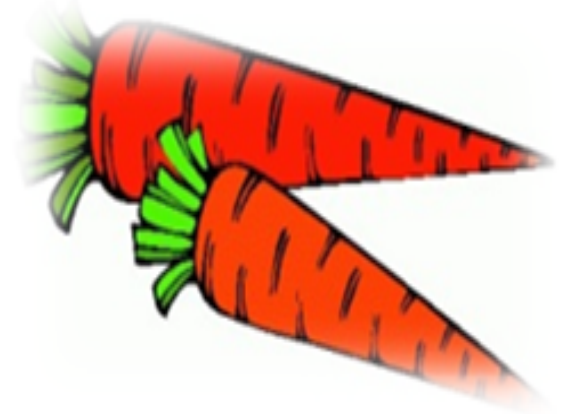

Powerful anti-oxidants vitamin C\&E: During this current pandemic when COVID-19 is so viral we might be thinking of taking Supplements of these two powerful antioxidants to fight against this viral infection. You will be surprised to know that consuming only 1 guava or strawberries or oranges or kiwi can fulfill your daily requirements of vitamin C.So what about Vitamin E? Here in Asia most of the people use Canola Oil for cooking their foods and most of the nuts and seeds contain a lot of vitamin E so a handful of nuts can do wonders.

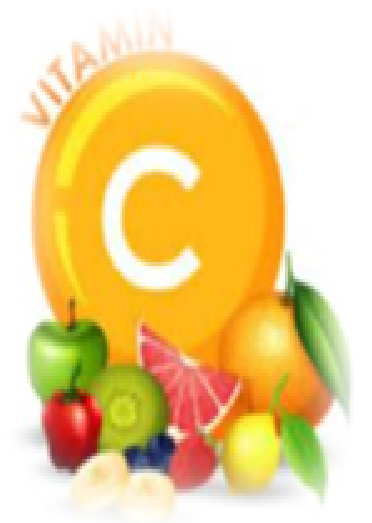

Volume 10 Issue 5 - 2020

\author{
Mahira Khan \\ Bachelors in Nutrition, Ward dietitian at Darulsehat hospital, \\ Pakistan
}

Correspondence: Mahira Khan, Bachelors in Nutrition, Ward dietitian at Darulsehat hospital, Flat G-2|4, Gulshan-e-shamim, Karachi, Pakistan, Email mahirakhan93@gmail.com

Received: August 16, 2020 | Published: October 21, 2020

Sunshine vitamin D: So why is vitamin D needed when we all know that it is related to bone health? You will be surprised to know that deficiency in vitamin $\mathrm{D}$ is related to susceptibility to infections. This is the only Vitamin which cannot be completed by DIET ALONE. Regular sun exposure is only natural way to get vitamin D. To maintain healthy blood levels, aim to get 10-30 minutes of midday sunlight, several times per week. If you are already deficient you might need supplements. Always check your Vitamin D levels before taking its Supplement.

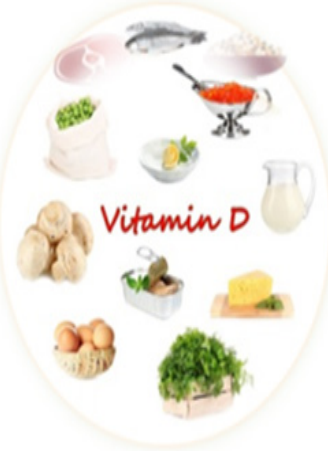

\section{Iron \& folate works together}

These two nutrients work together to fight against infections. The daily requirements of these nutrients can easily be met through diet.An intake of 1 cup of boiled beans or chickpeas or lentils in combination with palm-size meat (beef or mutton) can increase the absorption of iron and fulfill its requirements. Include 1 cup of most of the dark green leafy vegetables to fulfill folate requirements.

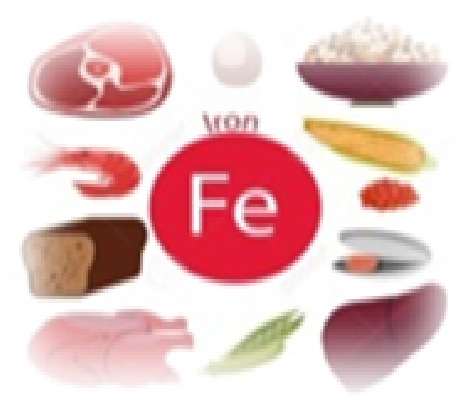


TIP: Include vitamin $\mathrm{C}$ rich foods along with iron to increase its absorption and avoid taking tea or coffee along with meals.

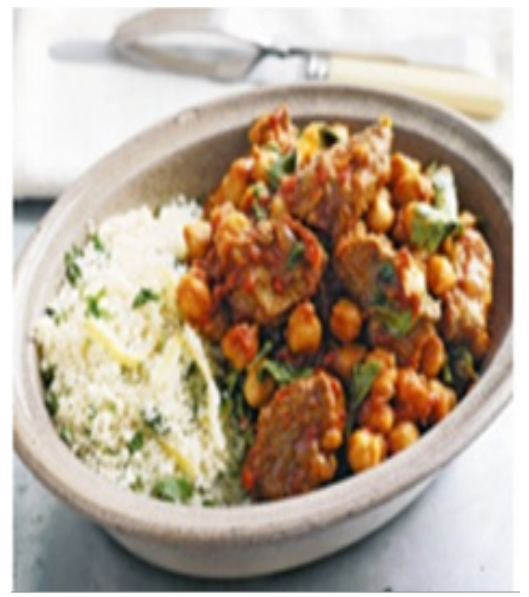

The trace minerals: Zinc, selenium and Copper are considered as the trace minerals because you need very little amount of these nutrients which can easily be met by taking variety of food items every day. Only taking 1cup of boiled chickpeas can fulfill copper requirements, 1 cup of boiled rice can fulfill selenium requirements and palm-sized beef can fulfill zinc requirements.

Vitamin B6 and B12: The body needs B6 in order to absorb vitamin B12 and to make red blood cells and cells of the immune system. You will be amazed that consuming only one small banana for vitamin B6 \& a cup of yoghurt for vitamin B12 is enough to meet their requirements.

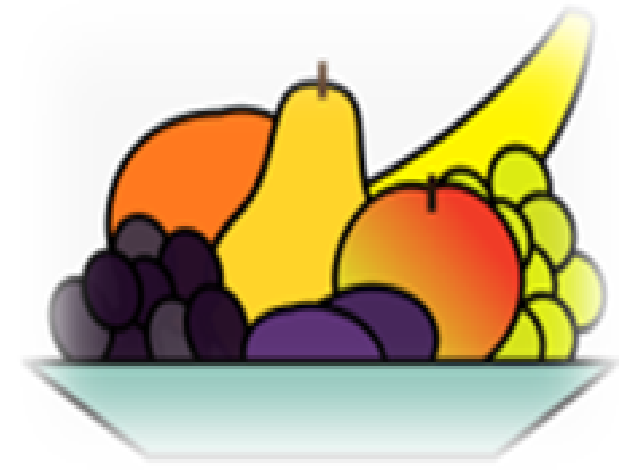

\section{Acknowledgments}

None.

\section{Conflicts of interest}

Author declare that there is no conflict of interest.

\section{Funding}

None. 